\title{
The stream fish fauna from three regions of the Upper Paraná River basin
}

\author{
Daniela Cristina Fagundes ${ }^{1,3}$, Cecilia Gontijo Leal ${ }^{I}$, Débora Reis de Carvalho ${ }^{I}$, Nara Tadini Junqueira ${ }^{I}$, \\ Francisco Langeani ${ }^{2}$ \& Paulo dos Santos Pompeu ${ }^{I}$ \\ ${ }^{1}$ Departamento de Biologia, Universidade Federal de Lavras, Lavras, MG, Brazil. \\ ${ }^{2}$ Universidade Estadual Paulista Júlio de Mesquita Filho, Departamento de Zoologia e Botânica, \\ São José do Rio Preto, SP, Brazil. \\ ${ }^{3}$ Corresponding author: Daniela Cristina Fagundes, e-mail: danielafagundes87@gmail.com
}

FAGUNDES, D.C., LEAL, C.G., CARVAlHO, D.R., JUNQUEIRA, N.T., LANGEANI, F., POMPEU, P.S. The stream fish fauna from three regions of the Upper Paraná River basin. Biota Neotropica. 15(2): e20140187. http://dx.doi.org/10.1590/1676-06032015018714

\begin{abstract}
This study aimed to conduct an inventory of fish assemblages in small streams that are tributaries of large reservoirs in three previously unstudied regions of the Upper Paraná River basin. We sampled 117 streams from $1^{\text {st }}$ to $3^{\text {rd }}$ order in Araguari (Nova Ponte hydropower plant reservoir), Paranaíba (São Simão reservoir) and Grande (Volta Grande reservoir) drainages. In total, 20,696 specimens belonging to 100 species, 53 genera, 20 families and six orders were collected. Of these, 11,530 specimens and 41 species were recorded in Araguari, whereas 17 were exclusive to this drainage. In Grande drainage, 3,537 individuals belonging to 41 species (11 exclusive) and in Paranaíba, 5,629 specimens and 67 species (38 exclusive) were sampled. The mean richness per stream was 6.7 for Grande, 9.0 for Araguari and 10.9 for Paranaíba drainage. The predominant orders were Characiformes, $48 \%$ of the total richness, and Siluriformes, 36\%. Three species were diagnosed as new: Astyanax sp. 1 (Grande and Paranaíba), Astyanax sp. 2 and Astyanax sp. 3 (both from Araguari), all from scabripinnis group. Another three were considered potentially new: Characidium sp. 1, Characidium sp. 2 and Rhamdiopsis sp. Among all records, eight species are not native from the Paraná basin: the exotics Oreochromis niloticus and Tilapia rendalli and the allochtonous Cyphocharax gillii, Hoplerythrinus unitaeniatus, Knodus moenkhausii, Poecilia reticulata, Roeboides descalvadensis and Trichomycterus brasiliensis. This study fills part of the existing knowledge gap about fish from small streams of the Paraná basin. Inventories of the fish fauna from these low-order water courses are important because they highlight their biodiversity relevance, including a significant number of unknown species. Thus, this study contributes to a better knowledge of the stream fish fauna of the upper Paraná River basin, as well as contribute to the establishment of strategies for conservation of this important component of aquatic biodiversity.
\end{abstract}

Keywords: fish, species inventory, new species.

FAGUNDES, D.C., LEAL, C.G., CARVAlHO, D.R., JUNQUEIRA, N.T., LANGEANI, F., POMPEU, P.S. A ictiofauna de riachos de três regiões do alto rio Paraná. Biota Neotropica. 15(2): e20140187. http://dx.doi.org/10.1590/1676-06032015018714

Resumo: Este trabalho teve como objetivo realizar um inventário das assembleias de peixes em pequenos riachos afluentes de grandes reservatórios do alto rio Paraná, em três regiões até então desconhecidas pela literatura. Foram amostrados 117 riachos de $1^{\mathrm{a}}$ a $3^{\mathrm{a}}$ ordens nas bacias dos rios Araguari (na bacia de drenagem do reservatório de Nova Ponte), Paranaíba (reservatório de São Simão) e Grande (reservatório de Volta Grande). No total foram capturados 20.696 exemplares pertencentes a 100 espécies, seis ordens, 20 famílias e 53 gêneros. Destes, 11.530 exemplares e 41 espécies foram registradas na bacia do Araguari, sendo que 17 dessas espécies são exclusivas. Na bacia do rio Grande, foram registrados 3.537 exemplares pertencentes a 41 espécies (11 exclusivas) e na bacia do Paranaíba, foram registrados 5.629 exemplares e 67 espécies (38 exclusivas). A riqueza média por riacho foi de 6.7 para os riachos da bacia do rio Grande, 9.0 para os riachos da bacia do Araguari e 10.9 para os riachos da bacia do rio Paranaíba. As ordens predominantes foram Characiformes, com $48 \%$ da riqueza total e Siluriformes, com $36 \%$. Três espécies pertencentes ao grupo scabripinnis foram diagnosticadas como novas: Astyanax sp. 1 (Grande e Paranaíba), Astyanax sp. 2 (Araguari) e Astyanax sp. 3 (Araguari). Outras três foram consideradas como potencialmente novas: Characidium sp. 1, Characidium sp. 2 e Rhamdiopsis sp. Entre todos os registros, oito espécies não são nativas da bacia do Paraná: as exóticas 
Oreochromis niloticus e Tilapia rendalli e as alóctones Cyphocharax gillii, Hoplerythrinus unitaeniatus, Knodus moenkhausii, Poecilia reticulata, Roeboides descalvadensis e Trichomycterus brasiliensis. Este estudo preenche parte da lacuna de conhecimento existente sobre peixes de pequenos riachos da bacia do Paraná. Os inventários sobre a fauna de peixes em riachos de pequena ordem são importantes, pois evidenciam a ocorrência de grande diversidade, incluindo um número significativo de espécies ainda não descritas. Dessa forma, esse estudo contribui para um melhor conhecimento da fauna de peixes de riachos pertencentes à bacia do alto rio Paraná, além de colaborar para o estabelecimento de estratégias para conservação deste importante componente da biodiversidade aquática.

Palavras-chave: peixes, inventário, espécie nova.

\section{Introduction}

Vertebrates are represented by approximately 55,000 species of which more than 31,000 are fish (IUCN 2008; Froese \& Pauly 2009; Eschmeyer \& Fong 2010). Neotropical freshwater ecosystems encompass a great part of this diversity, namely 4,475 valid species and about other 1,550 yet to be described (Reis et al. 2003). Therefore this region is considered to have the most diverse freshwater fish fauna in the world (Junk 2007). Brazil covers most of the Neotropical region, and its' ichthyofauna hosts more than 3,000 freshwater species (Froese \& Pauly 2014). This is due to, among other factors, the country's vast extension and complex hydrological network including some of the largest river drainages in the world.

The Paraná River basin is the second largest in drainage area in Brazil (Latrubesse et al. 2005). The upper portion of the basin is considered the best studied region in terms of its ichthyofauna, however this knowledge is still insufficient and several regions remain completely unknown. Therefore the present study aimed to present the results of an inventory conducted in small streams draining to three large reservoirs in the Upper Paraná, in areas thus far unstudied. The information presented here can be used to inform management and conservation strategies.

\section{Materials and Methods}

\section{Study Area}

The Upper Paraná River encompasses the Paraná River basin upstream the former Salto de Sete Quedas (Bonetto 1986). This portion of the basin represents $891.000 \mathrm{~km}^{2}(10.5 \%$ of Brazil's total area) (Agostinho et al. 2003) and includes important tributaries such as the Araguari, Grande, Paranaíba, Paranapanema and Tietê, and the first three were included in the present study. The Araguari River is a left bank tributary of the Paranaíba River and has its headwaters in the Parque Nacional da Serra da Canastra, São Roque de Minas municipality, Minas Gerais state. This tributary is $475 \mathrm{~km}$ of length and covers $21,856 \mathrm{~km}^{2}$ of drainage area (Baccaro et al. 2004). The headwaters of the Grande River are in the Serra da Mantiqueira, Bocaina de Minas municipality. Approximately $86,500 \mathrm{~km}^{2}$ of its total $143,000 \mathrm{~km}^{2}$ drainage area is within Minas Gerais state limits. The Paranaíba has its headwaters in the Serra da Mata da Corda, 1,100 m above sea level and its drainage area encompasses $222,711 \mathrm{~km}^{2}$, with ca $30 \%$ in Minas Gerais state. Together, the rivers Paranaíba and Grande form the Paraná River.

This study was carried out in 117 streams of $1^{\text {st }}$ to $3^{\text {rd }}$ order (according to Sthraler 1957) belonging to Araguari

(38 streams), Paranaíba (39 streams) and Grande river (40 streams) drainages (Figure 1).
1.1. Fish Sampling and Identification. Stream sampling was carried out during the dry season, in September 2009 (Araguari), 2011 (Grande), 2012 (Paranaíba) and 2013 (resampling of Araguari streams). The length of each stream site sampled was 40 times its mean width, with a minimum length of $150 \mathrm{~m}$ (Kaufmann et al. 1999). Each stream was sampled for two hours by three people using mainly semicircular hand nets ( $80 \mathrm{~cm}$ of diameter, $1 \mathrm{~mm}$ of mesh size) and seines when possible ( $4 \mathrm{~m}$ long, $2 \mathrm{~m}$ high, $5 \mathrm{~mm}$ of mesh size). All catches were made during daylight hours. Specimens were killed in an anesthetic solution of clove oil and then fixed in $10 \%$ formalin. In the laboratory, all sampled fishes were transferred to $70^{\circ} \mathrm{GL}$ alcohol and identified to species level, by means of identification key (Graça \& Pavanelli 2007) and expert (Francisco Langeani). Voucher specimens of all species are deposited at the following fish collections: Coleção de Ictiologia da Universidade Federal de Lavras (CIUFLA), Coleção do Departamento de Zoologia da Universidade Estadual Paulista, São José do Rio Preto campus (DZSJRP), Museu de Ciências e Tecnologia da PUCRS (MCP), Museu de Zoologia da Universidade de São Paulo (MZUSP), Museu Paraense Emilio Goeldi (MPEG) and Coleção Ictiológica do Nupélia (NUP), Universidade Estadual de Maringá (Table 1).

\section{Results and Discussion}

In total we collected 20,696 specimens belonging to 100 species, 53 genus, 20 families and six orders. Araguari accounted for 11,530 specimens and 41 species whereas 17 species were exclusive to this drainage. Grande drainage was represented by 3,537 individuals belonging to 41 species (11 exclusive) and the Paranaíba by 5,629 specimens and 67 species (38 exclusive). Paranaíba showed the highest mean richness per stream, 10.9, followed by Araguari, 9.0 and Grande, 6.7 (Table 1)

Characiformes and Siluriformes orders represented the majority of the species richness, $48 \%$ and 36\% respectively, reflecting a well known pattern recognized for South American rivers (Lowe-McConnell 1987). Langeani et al. (2007) recorded 310 species included in 11 orders for the Upper Paraná basin, with $80 \%$ represented by Characiformes and Siluriformes, a number similar to our findings. Likewise Alves et al. (2007) found a predominance of Characiformes (47\%) and Siluriformes $(32 \%)$ in the Minas Gerais portion of the Upper Paraná basin.

At the family level, the Characidae was the best represented in this study. Other studies also found the same pattern (Castro et al. 2003; 2004; Shibatta et al. 2007). The Characidae is one of the richest families among the Neotropical freshwater fish fauna (Reis et al. 2003), and present a wide range of feeding habits and habitat uses (Kavalco \& Pazza 2007). 
Fish, Species inventory, New species

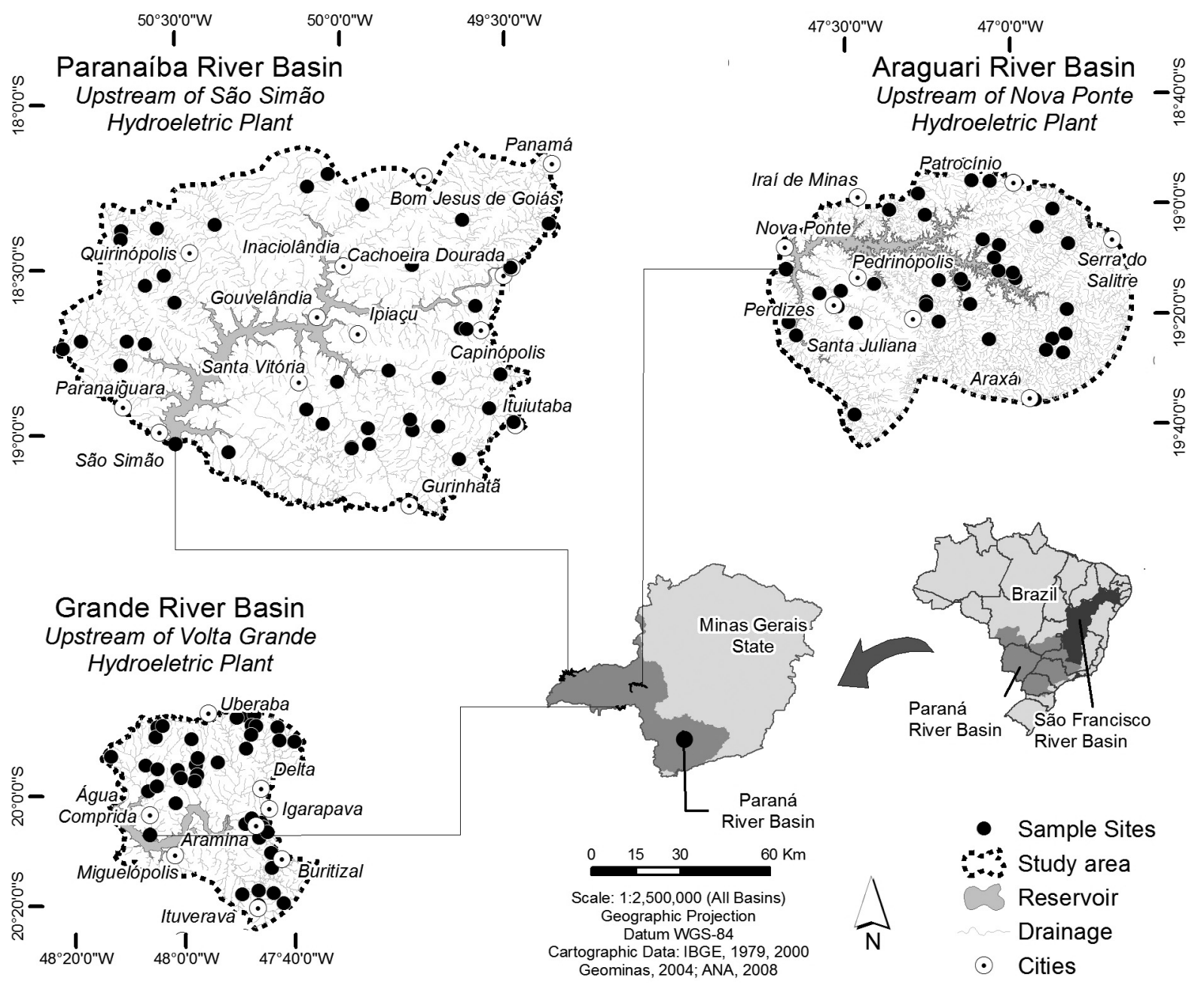

Figure 1. Map of the study area showing the location of the 117 streams in the Upper Paraná River basin.

The Astyanax scabripinnis group is particularly abundant and widespread in small streams in several Brazilian river basins (Bertaco \& Lucena 2006), including the Upper Paraná River basin (Castro \& Casatti 1997; Pavanelli \& Caramaschi 1997). In the present study three species within the scabripinnis group were identified as probably new: Astyanax sp. 1 (Grande and Paranaíba), Astyanax sp. 2 (Araguari) and Astyanax sp. 3 (Araguari). Besides these, another three species were considered potentially new: Characidium sp. 1, Characidium sp. 2 e Rhamdiopsis sp.

Besides being a well studied region, the Upper Paraná still hosts several new species, some of them recently discovered and described. Langeani et al. (2007) listed 50 new species for the region, whereas 16 were formally described since then and another 34 await the same process (Leporinus piavussu Britski, Birindelli \& Garavello 2012; Astyanax bockmanni Vari \& Castro 2007; Hemigrammus parana Marinho, Carvalho, Langeani \& Tatsumi 2008; Characidium heirmostigmata Graça \& Pavanelli 2008; Characidium xanthopterum Silveira, Langeani, Graça, Pavanelli \& Buckup 2008; Apteronotus acidops Triques 2011; Gymnorhamphichthys britskii Carvalho, Ramos \& Albert 2011; Rhinolekos britskii Martins, Langeani \& Britskii 2011; Hypostomus multidens Jerep, Shibatta \& Zawadzki 2007; Harttia absaberi Oyakawa, Fichberg \& Langeani 2013; Neoplecostomus corumba Zawadzki, Pavanelli
\& Langeani 2008; Neoplecostomus selenae Zawadzki, Pavanelli \& Langeani 2008; Neoplecostomus yapo Zawadzki, Pavanelli \& Langeani 2008; Phalloceros harpagos Lucinda 2008; Phalloceros reisi Lucinda 2008; Laetacara araguaiae Ottoni \& Costa 2009). In addition to the new species mentioned by Langeani et al. (2007), another 29 were also described for the region (Hyphessobrycon uaiso Carvalho \& Langeani 2013; Hyphessobrycon vinaceus Betarco, Malabarba \& Dergam 2007; Corumbataia britskii Ferreira \& Ribeiro 2007; Hisonotus piracanjuba Martins \& Langeani 2012; Microlepidogaster dimorpha Martins \& Langeani 2011; Microlepidogaster arachas Martins, Calegari \& Langeani 2012; Microlepidogaster longicolla Calegari \& Reis 2010; Otothyropsis biamnicus Calegari, Lehmann \& Reis 2013; Otothyropsis Polyodon Calegari, Lehmann \& Reis 2013; Rhinolekos schaeferi Martins \& Langeani 2011; Rhinolekos garavelloi Martins \& Langeani 2011; Hypostomus denticulatus Zawadzki, Weber \& Pavanelli 2008; Hypostomus heraldoi Zawadzki, Weber \& Pavanelli 2008; Neoplecostomus bandeirante Roxo, Oliveira \& Zawadzki 2012; Neoplecostomus botucatu Roxo, Oliveira \& Zawadzki 2012; Neoplecostomus langeanii Roxo, Oliveira \& Zawadzki 2012; Iheringichthys syi Azpelicueta \& Britskii 2012; Trichomycterus perkos Datovo, Carvalho \& Ferrer 2012; Trichomycterus pirabitira Barbosa \& Azevedo Santos 2012; Trichomycterus piratymbara Katz, Barbosa \& Costa 2013; Trichomycterus septemradiatus Katz, 
Table 1. Fish species sampled in Upper Paraná River basin streams. $\mathrm{N}=$ number of individuals, CI-UFLA $=$ voucher specimens deposited at Coleção de Peixes da Universidade Federal de Lavras (UFLA), DZSJRP = Coleção do Departamento de Zoologia de São José do Rio Preto, MCP = Museu de Ciências e Tecnologia da PUCRS, MPEG = Museu Paraense Emilio Goeldi, MZUSP = Museu de Zoologia da Universidade de São Paulo, NUP $=$ Coleção ictiológica do Nupélia. ${ }^{*}=$ allochtonous species, ${ }^{* *}=$ exotic species, ${ }^{* * *}=$ migratory species, ${ }^{* * * *}=$ probably nem species, $X=$ specimens that couldn't be deposited in a scientific collection because they were used in stable isotopes study, in this case they were identified through photos taken in the field.

\begin{tabular}{lll}
\hline TAXON (Catalog of Fishes) & Upper Paraná River basin Collection & Voucher \\
\cline { 2 - 2 } CHARACIFORMES & Araguari Grande Paranaíba
\end{tabular}

Acestrorhynchidade

Acestrorhynchus lacustris (Lütken, 1875)

Anostomidae

Leporinus friderici (Bloch, 1794) ***

Leporinus lacustris Amaral Campos, 1945

Leporinus microphthalmus Garavello, 1989

Leporinus octofasciatus Steindachner, 1915

Leporinus paranensis Garavello \& Britski, 1987

Leporinus piavussu Britski, Birindelli \&

Garavello, 2012

Leporinus striatus Kner, 1858

Characidae

Aphyocharax dentatus Eigenmann \& Kennedy, 1903

Astyanax altiparanae Garutti \& Britski, 2000

Astyanax bockmanni Vari \& Castro, 2007

Astyanax fasciatus (Cuvier, 1819)

Astyanax sp. 1****

Astyanax sp. 2****

Astyanax sp. 3****

Bryconamericus cf. stramineus Eigenmann, 1908

Bryconamericus stramineus Eigenmann, 1908

Bryconamericus turiuba Langeani, Lucena,

Pedrini \& Tarelho-Pereira, 2005

Brycon orbignyanus (Valenciennes, 1850) ***

Glandulocaudinae sp.

Hasemania sp.

Hemigrammus parana Marinho, Carvalho,

Langeani \& Tatsumi, 2008

Hyphessobrycon eques (Steindachner, 1882)

Knodus moenkhausii (Eigenmann \& Kennedy, 1903)*

Moenkhausia intermedia Eigenmann, 1908

Moenkhausia sanctaefilomenae (Steindachner, 1907)

Oligosarcus cf. planaltinae

Oligosarcus pintoi Amaral Campos, 1945

Piabina argentea Reinhardt, 1867

Roeboides descalvadensis Fowler, 1932*

Serrapinus sp. 1

Serrapinus sp. 2

Triportheus nematurus (Kner, 1858)

Crenuchidae

Characidium cf. zebra Eigenmann, 1909

Characidium gomesi Travassos, 1956

Characidium sp. $1 * * * *$

Characidium sp. 2****

Characidium xanthopterum Silveira, Langeani,

da Graça, Pavanelli \& Buckup, 2008

\section{1}

$-\quad-\quad-10$

$-$

26

$-$$$
-
$$

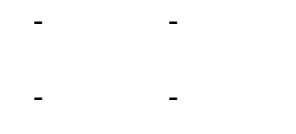

44

3

1002$$
61
$$

61

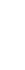

1

2

308

$$
52
$$

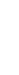$$
6
$$$$
142
$$$$
29
$$$$
67
$$

156

315

192

-

$-$

$-$

4$$
-
$$

2

5

5

6
CIUFLA $\quad 0464 / 0769 / 0749 / 0807$
CIUFLA 0750/0808

CIUFLA 0770/0751/0809

CIUFLA $0752 / 0810$

CIUFLA 0463/0771

CIUFLA 0465/0772

CIUFLA 0881

CIUFLA 0467/0773/0754/0812

CIUFLA 0774/0755

CIUFLA 0753/0811

MZUSP 114314

CIUFLA 0783/822

CIUFLA 0784

8

2517

CIUFLA 0826

CIUFLA 0481/0789/

CIUFLA 0734/0839

1 CIUFLA 0845

14 CIUFLA 0846

$-$

145

CIUFLA 0486/0792

CIUFLA 0736

CIUFLA 0489/0795/0738/0849

DZSJRP 20030/20031

5

CIUFLA

0855

40

127

CIUFLA

DZSJRP

CIUFLA

DZS

$\begin{array}{lll}- & - & 12\end{array}$

$4 \quad-\quad-$

$30 \quad 41 \quad 45$

- $76 \quad-$

$141 \quad-\quad-$

$20-$
CIUFLA 0858

CIUFLA 0776

CIUFLA 0777/0757/0814

CIUFLA 0863

CIUFLA 0469/0778

CIUFLA 0471 
Table 1. Continued.

\begin{tabular}{|c|c|c|c|c|c|}
\hline \multirow{2}{*}{$\begin{array}{l}\text { TAXON (Catalog of Fishes) } \\
\text { CHARACIFORMES }\end{array}$} & \multicolumn{3}{|c|}{ Upper Paraná River basin } & \multirow[t]{2}{*}{ Collection } & \multirow[t]{2}{*}{ Voucher } \\
\hline & Araguari & Grande & Paranaíba & & \\
\hline Characidium zebra Eigenmann, 1909 & - & - & 306 & CIUFLA & 0815 \\
\hline \multicolumn{6}{|l|}{ Curimatidae } \\
\hline Cyphocharax gilli Eigenmann \& Kennedy, 1903* & - & - & 4 & & $\mathrm{X}$ \\
\hline Steindachnerina insculpta (Fernández-Yépez, 1948) & - & - & 10 & CIUFLA & 0856 \\
\hline \multicolumn{6}{|l|}{ Erythrinidae } \\
\hline Hoplerythrinus unitaeniatus (Spix \& Agassiz, 1829)* & - & 1 & - & CIUFLA & 0766 \\
\hline Hoplias aff. malabaricus (Bloch, 1794) & 6 & - & 1 & CIUFLA & $0476 / 0785 / 0825$ \\
\hline Hoplias intermedius (Günther, 1864) & 3 & 3 & 3 & CIUFLA & $0475 / 0763 / 0824$ \\
\hline \multicolumn{6}{|l|}{ Lebiasinidae } \\
\hline Pyrrhulina australis Eigenmann \& Kennedy, 1903 & - & 12 & 4 & CIUFLA & $0740 / 0852$ \\
\hline \multicolumn{6}{|l|}{ Parodontidade } \\
\hline Apareiodon cf. piracicabae Eigenmann, 1907 & - & - & 5 & CIUFLA & 0804 \\
\hline Apareiodon ibitiensis Amaral Campos, 1944 & 158 & 32 & 119 & CIUFLA & 0461/0768/0747/0805 \\
\hline Parodon nasus Kner, 1859 & 6 & - & - & CIUFLA & $0487 / 0793$ \\
\hline \multicolumn{6}{|l|}{ CYPRINODONTIFORMES } \\
\hline \multicolumn{6}{|l|}{ Poeciliidae } \\
\hline Phalloceros harpagos Lucinda, 2008 & 2017 & 2 & - & CIUFLA & 0488/0794/0737 \\
\hline Poecilia reticulata Peters, 1859* & 43 & 532 & 112 & CIUFLA & 0490/0796/0739/0851 \\
\hline \multicolumn{6}{|l|}{ Rivulidae } \\
\hline Rivulus apiamici Costa, 1989 & - & - & 11 & CIUFLA & 0854 \\
\hline \multicolumn{6}{|l|}{ GYMNOTIFORMES } \\
\hline \multicolumn{6}{|l|}{ Gymnotidae } \\
\hline Gymnotus carapo Linnaeus, 1758 & - & - & 1 & CIUFLA & 0820 \\
\hline Gymnotus cf. inaequilabiatus Valenciennes, 1839 & - & 1 & - & CIUFLA & 0760 \\
\hline Gymnotus sylvius Albert \& Fernandes-Matioli, 1999 & 16 & 16 & 3 & CIUFLA & 0474/0782/0761/0821 \\
\hline \multicolumn{6}{|l|}{ Sternopygidae } \\
\hline Eigenmannia trilineata López \& Castello, 1966 & - & - & 7 & CIUFLA & 0818 \\
\hline Eigenmannia virescens (Valenciennes, 1836) & - & - & 3 & CIUFLA & 0819 \\
\hline Sternopygus macrurus (Bloch \& Schneider, 1801) & - & - & 1 & & $\mathrm{X}$ \\
\hline \multicolumn{6}{|l|}{ PERCIFORMES } \\
\hline \multicolumn{6}{|l|}{ Cichlidae } \\
\hline Cichlasoma paranaense Kullander, 1983 & 9 & 2 & 10 & CIUFLA & $0779 / 0765 / 0816$ \\
\hline Crenicichla jaguarensis Haseman, 1911 & - & - & 2 & CIUFLA & 0817 \\
\hline Geophagus brasiliensis (Quoy \& Gaimard, 1824) & 47 & 13 & - & CIUFLA & 0473/0781/0759 \\
\hline Laetacara araguaiae Ottoni \& Costa, 2009 & - & - & 29 & CIUFLA & 0840 \\
\hline Oreochromis niloticus (Linnaeus, 1758) ** & - & - & 81 & CIUFLA & 0847 \\
\hline Tilapia rendalli (Boulenger, 1897) ** & - & 1 & - & CIUFLA & 0744 \\
\hline \multicolumn{6}{|l|}{ SILURIFORMES } \\
\hline \multicolumn{6}{|l|}{ Auchenipteridae } \\
\hline Tatia neivai (Ihering, 1930) & - & 1 & 1 & CIUFLA & 0764/X \\
\hline Callichthydae & & & & & \\
\hline Aspidoras fuscoguttatus Nijssen \& Isbrücker, 1976 & - & 22 & 208 & CIUFLA & $0748 / 0806$ \\
\hline Corydoras difluviatilis Britto \& Castro, 2002 & 70 & 2 & - & CIUFLA & $0472 / 0780 / 0758$ \\
\hline Cetopsidae & & & & & \\
\hline Cetopsis gobioides Kner, 1858 & - & - & 1 & & $\mathrm{X}$ \\
\hline Heptapteridae & & & & & \\
\hline Cetopsorhamdia iheringi Schubart \& Gomes, 1959 & 45 & 60 & 20 & CIUFLA & 0468/0775/0756/0813 \\
\hline Imparfinis borodini Mees \& Cala, 1989 & - & 3 & 7 & CIUFLA & $0732 / 0836$ \\
\hline Imparfinis cf. schubarti Gomes, 1956 & - & - & 2 & CIUFLA & 0837 \\
\hline Imparfinis longicauda (Borodin, 1927) & - & - & 5 & & $\mathrm{X}$ \\
\hline Imparfinis schubarti (Gomes, 1956) & 3 & 11 & 65 & CIUFLA & 0480/0788/0733/0838 \\
\hline Phenacorhamdia cf. unifasciata Britiski, 1996 & - & - & 8 & CIUFLA & 0848 \\
\hline Pimelodella gracilis (Valenciennes, 1835) & - & - & 36 & CIUFLA & 0850 \\
\hline Rhamdia quelen (Quoy \& Gaimard, 1824) & 37 & 44 & 21 & CIUFLA & 0491/0797/0741/0853 \\
\hline Rhamdiopsis sp. **** & 72 & 13 & - & CIUFLA & 0479/0798/0742 \\
\hline
\end{tabular}


Table 1. Continued.

\begin{tabular}{|c|c|c|c|c|c|}
\hline \multirow{2}{*}{$\begin{array}{l}\text { TAXON (Catalog of Fishes) } \\
\text { CHARACIFORMES }\end{array}$} & \multicolumn{3}{|c|}{ Upper Paraná River basin } & \multirow[t]{2}{*}{ Collection } & \multirow[t]{2}{*}{ Voucher } \\
\hline & Araguari & Grande & Paranaíba & & \\
\hline \multicolumn{6}{|l|}{ Loricariidae } \\
\hline Hisonotus piracanjuba Martins \& Langeani, 2012 & - & - & 183 & CIUFLA & 0823 \\
\hline \multirow[t]{2}{*}{ Hisonotus francirochai (Ihering, 1928) } & - & 98 & - & CIUFLA & 0762 \\
\hline & & & & MUP & $\begin{array}{l}48252 / 48253 / 48254 / \\
48255 / \\
48256 / 48257 / 48258 / \\
48259\end{array}$ \\
\hline \multirow[t]{2}{*}{ Hypostomus aff. nigromaculatus Schubart, 1964} & - & 235 & 89 & CIUFLA & $0864 / 0834$ \\
\hline & & & & NUP & $\begin{array}{l}16115 / 16117 / 16126 / \\
16130 / 16132\end{array}$ \\
\hline \multirow[t]{2}{*}{ Hypostomus ancistroides (Ihering, 1911) } & - & 499 & 59 & CIUFLA & $0865 / 0827$ \\
\hline & & & & NUP & $\begin{array}{l}16426 / 16427 / 16428 / \\
16429 / 16430 / 16431 / \\
16432 / 16434 / \\
16435 / 16436 / 16437 / \\
16438\end{array}$ \\
\hline Hypostomus cf. paulinus Ihering, 1905 & - & - & 56 & CIUFLA & 0828 \\
\hline Hypostomus cf. topavae Godoy, 1969 & - & - & 45 & CIUFLA & 0829 \\
\hline Hypostomus nigromaculatus (Schubart, 1964) & - & 198 & - & NUP & $\begin{array}{l}16112 / 16113 / 16114 / \\
16116 / 16118 / 16124 / \\
16128 / 16129 / 16131 / \\
16134 / 16135 / 16136\end{array}$ \\
\hline Hypostomus sp. 1 & 741 & - & - & CIUFLA & $0477 / 0786$ \\
\hline Hypostomus sp. 2 & 109 & - & - & CIUFLA & $0478 / 0787$ \\
\hline Hypostomus sp. 3 & - & - & 82 & CIUFLA & 0830 \\
\hline Hypostomus sp. 4 & - & - & 148 & CIUFLA & 0831 \\
\hline Hypostomus sp. 5 & - & - & 94 & CIUFLA & 0832 \\
\hline Hypostomus sp. 6 & - & - & 26 & CIUFLA & 0833 \\
\hline \multirow[t]{2}{*}{ Hypostomus strigaticeps (Regan, 1908) } & - & 6 & 26 & CIUFLA & 0835 \\
\hline & & & & NUP & $\begin{array}{l}16123 / 16127 / 16133 / \\
16433\end{array}$ \\
\hline $\begin{array}{l}\text { Microlepidogaster arachas Martins, Calegari \& } \\
\text { Langeani, } 2013\end{array}$ & 512 & - & - & CIUFLA & $0484 / 0790$ \\
\hline Neoplecostomus paranensis Langeani, 1990 & - & 13 & - & CIUFLA & 0735 \\
\hline Neoplecostomus sp. & 79 & - & - & CIUFLA & $0485 / 0791$ \\
\hline \multicolumn{6}{|l|}{ Trichomycteridae } \\
\hline \multirow[t]{2}{*}{ Trichomycterus aff. brasiliensis Lütken, 1874} & - & 176 & - & CIUFLA & 0745 \\
\hline & & & & MPEG & 24986 \\
\hline Trichomycterus brasiliensis Lütken, 1874* & 258 & - & - & CIUFLA & 0800 \\
\hline \multirow[t]{2}{*}{ Trichomycterus candidus (Miranda Ribeiro, 1949) } & - & 284 & - & CIUFLA & 0746 \\
\hline & & & & MPEG & 24979 \\
\hline Trichomycterus sp. 1 & 1418 & - & - & CIUFLA & 0493/0801 \\
\hline Trichomycterus sp. 2 & 177 & - & - & CIUFLA & $0732 / 0802$ \\
\hline Trichomycterus sp. 3 & 2 & - & - & CIUFLA & 0803 \\
\hline \multicolumn{6}{|l|}{ SYNBRANCHIFORMES } \\
\hline \multicolumn{6}{|l|}{ Synbranchidae } \\
\hline Synbranchus marmoratus Bloch, 1795 & 3 & 3 & 4 & CIUFLA & 0492/0799/0743/0857 \\
\hline Total abundance & 11530 & 3537 & 5629 & & \\
\hline Total richness & 41 & 41 & 67 & & \\
\hline Mean richness per stream & 8.97 & 6.73 & 10.85 & & \\
\hline Exclusive species & 17 & 11 & 38 & & \\
\hline
\end{tabular}

Barbosa \& Costa 2013; Pituna brevirostrata Costa 2007; Melanorivulus faucireticulatus Costa 2007; Melanorivulus illuminatus Costa 2007; Rivulus giarettai Costa 2008; Rivulus formosensis
Costa 2008; Simpsonichthys margaritatus Costa 2011; Simpsonichthys nigromaculatus Costa 2007; Australoheros tavaresi Ottoni 2012 and Pimelodus britskii Garavello \& Shibatta 2007. 
Among all recorded species, six are considered allochtonous, i.e. native to other Neotropical river basins and introduced in the upper Paraná basin. Of these six species, Cyphocharax gillii and Roeboides descalvadensis were collected only in the Paranaíba basin, Hoplerythrinus unitaeniatus in the Grande basin and Trichomycterus brasiliensis in Araguari basin. On the other hand Poecilia reticulata and Knodus moenkhausii occurred in each of the drainages. The most abundant allochtonous species, $K$. moenkhausii (especially in the Paranaíba basin) is an opportunistic species that invests much of its energy in reproduction and colonize several environments, including the most degraded (Ceneviva-Bastos \& Casatti 2007). Poecilia reticulata (the second most abundant allochthonous species) is native to northwestern America (Bisazza 1993) and has been introduced in several regions of the world because of its potential for mosquito control (Araújo et al. 2009). This species also has great ability to increase its population in harsh environments, and is therefore indicative of poor water quality (Araújo 1983).

The two exotic species (i.e. native to other continents) recorded in our study area, Oreochromis niloticus and Tilapia rendalli occurred only in one of the studied drainages each, Grande and Paranaíba respectively. Both species are of medium size and have great commercial value, which probably motivated their introduction. Oreochromis niloticus is native to the Nile River basin, East Africa but is largely introduced in tropical and subtropical regions (Carvalho 2006). It was first introduced in northeast Brazil in the 1970s, through the Departamento Nacional de Obras Contra a Seca (DNOCS), and subsequently spread throughout the whole country (Castagnolli 1996). It is classified as a pest and its introduction has a well known negative impact to the aquatic ecosystems (Froese \& Pauly 2010).

Two migratory species were recorded, Brycon orbignyanus (piracanjuba) and Leporinus friderici (piau-três-pintas), both represented by few individuals. Migratory species in the adult stage are mostly residents of larger streams or rivers, being occasionally found in these low-order streams when in their young stage (Pompeu \& Godinho 2003). Brycon orbignyanus is considered endangered in Minas Gerais state, according to the IUCN Red Data List (Machado et al 2005; Alves et al 2007), due to habitat degradation especially driven by clearance of the riparian vegetation, pollution and construction of hydropower plants (Santos 2010).

This study fills part of the knowledge gap about fish from small streams of the Paraná basin by presenting the species list of regions unstudied until this moment. Inventories of the fish fauna from these low-order water courses are important because they highlight their biodiversity relevance, including a significant number of new species. As such, our findings can help inform future conservation and/or management strategies in the studied landscape.

\section{Acknowledgments}

The present study is part of the project: "Desenvolvimento de índices de integridade biótica (IBI em inglês) como ferramenta para avaliar aqualidade ambiental e subsidiar a restauração de habitats em áreas de soltura de alevinos pela CEMIG". We thank our colleagues at the Laboratório de Ecologia de Peixes (UFLA) and Laboratório de Ecologia de Bentos (UFMG) for their help with the field work, Claúdio
Zawadzki, Guilherme Dutra, Naércio Menezes, Roberto Reis and Wolmar Wosiacki for helping with the species identification, the landowners for providing permission to conduct research on their properties, IBAMA for the collecting license (10327-1). We also thank Companhia Energética de Minas Gerais (CEMIG), Fundação de Amparo à Pesquisa do Estado de Minas Gerais (FAPEMIG, CRA 03147/11), Coordenação de Aperfeiçoamento de Pessoal de Nível Superior (CAPES, CGL received scholarship: BEX2943/13-1) and P\&D ANEEL/ CEMIG GT 487 for financial support including the authors scholarships; FAPESP and CNPq for financial support to the studies of the ichthyofauna from the Upper Paraná and the modernization of the fish collection at DZSJRP (FL). PSP received a research fellowship from the Conselho Nacional de Desenvolvimento Científico e Tecnológico (CNPq No. 306325/ 2011-0) and from the Fundação de Amparo à Pesquisa do Estado de Minas Gerais (FAPEMIG No. PPM-00237/13).

\section{References}

AGOSTINHO, A.A., GOMES, L.C., SUZUKI, H.I., JULIO, Jr., H.F. 2003. Migratory fishes of the Upper Paraná river basin, Brazil. In CROSFELD, J., HARVEY, B., ROSS, C., BAER, A. Migratory Fishes of South America Victoria: world Fisheries Trust, p. 20-98.

AGOSTINHO, A.A., GOMES, L.C., PELICICE, F.M. 2007. Ecologia e manejo de recursos pesqueiros em reservatórios do Brasil. Maringá: Eduem, $501 \mathrm{p}$.

ALVES, C.B.M., VIEIRA, F., MAGALHÃES, A.L.B., BRITO, M.F. G. 2007. Impacts of non-native fish species in Minas Gerais, Brazil: presente situation and prospects. In BERT, M.T. Ecological and genetic implications of aquaculture activities, Dordrecht: Springer, p. 291-314.

ARAUJO, F.G., PEIXOTO, M.G., PINTO, B.C.T., TEIXEIRA, T.P. 2009. Distribution of guppies Poecilia reticulata (Peters, 1860) and Phalloceros caudimaculatus (Hensel, 1868) along a polluted stretch of the Paraíba do Sul River, Brazil. Braz. J. Biol. 69(1): 41-48

ARAUJO, J.R.S. 1983. Projeto de biodetecção de tóxicos em sistemas fluviais de utilização em captação de água para sistemas públicos de abastecimento. Cadernos FEEMA, Série Congressos 17(83): 1-129.

BACARRO, C.A., MEDEIROS, S.M., FERREIRA, I.L., RODRIGUES, S.C. 2004. Mapeamento Geomorfológico da Bacia do Rio Araguari (MG). LIMA, S.C. \& SANTOS, R.J. (Org.). In: Gestão Ambiental da Bacia do Rio Araguari - rumo ao desenvolvimentosustentável. Uberlandia: CNPq, p. 1-20.

BERTACO, V.A. \& LUCENA, C.A.S. 2006. Two new species of Astyanax (Ostariophysi: Characiformes: Characidae) from eastern Brazil, with a synopsis of the Astyanax scabripinnis species complex. Neotropical Ichthyology, 4(1):53-60.

BISAZZA, A., MANFREDI, S., PILASTRO, A. 2000. Sexual competition, coercive mating and mate assessment in the one-sided livebearer, Jenynsiamultidentata: are they predictive of sexual dimorphism? Ethology, 106(11):961-978.

BONETTO, A.A. 1986. The Paraná river system. In: DAVIES, B.R., WALKER, K.F. The ecology of river systems. Dordrecht: Dr. W. Junk Publishers, p. 541-555.

CARVALHO, E.D. 2006. Avaliação dos impactos da piscicultura em tanques-rede nas represas dos grandes tributários do alto Paraná (Tietê e Paranapanema): o pescado, a ictiofauna agregada e as condições limnológicas. Relatório Científico (FAPESP). Botucatu, SP. 46 p.

CASTAGNOLLI, N. 1996. Aqüicultura para o ano 2000. Brasília: CNPq, 95p.

CASTRO, R.M.C. \& CASATTI, L. 1997. The fish fauna from a small forest stream of the upper Paraná River basin, Southeastern 
Brasil. Ichthyological Exploration Freshwaters, Cornol, 7(4): 337-352.

CASTRO, R.M.C., CASATTI, L., SANTOS, H.F., FERREIRA, K. M., RIBEIRO, A.C., BENINE, R.C., DARDIS, G.Z.P., MELO, A.L.A., ABREU, T.X., BOCKMANN, F.A., CARVALHO, M., GIBRAN, F.Z., LIMA, F.C.T. 2003. Estrutura e composição da ictiofauna de riachos do Rio Paranapanema, sudeste e sul do Brasil. Biota Neotropica 3(1): http://www.biotaneotropica.org.br/ v3n1/pt/abstract?article+BN01703012003.

CASTRO, R.M.C., CASATTI, L., SANTOS, H.F., MELO, A.L.A., MARTINS, L.S.F., FERREIRA, K.M., GIBRAN, F.Z., BENINE, R.C., CARVALHO, M., RIBEIRO, A.C., ABREU, T. X., BOCKMANN, F.A., DARDIS, G.Z.P., STOPIGLIA, R., LANGEANI, F. 2004. Estrutura e composição da ictiofauna de riachos da bacia do Rio Grande, no Estado de São Paulo, Sudeste do Brasil. Biota Neotropica 4(1): http://www.biotaneotropica.org. br/v4n1/pt/abstract? article + BN0170402004.

CENEVIVA-BASTOS, M. \& CASATTI, L. 2007. Oportunismo alimentar de Knodus moenkhausii (Teleostei, Characidae): uma espécie abundante em riachos do noroeste do Estado de Sao Paulo, Brasil. Iheringia. Série Zoologia, 97:7-15.

ESCHMEYER, W.N. \& FONG, J.D. 2010. Species of Fishes by family/subfamily. Disponível em: http://research.calacademy.org/ research/ichthyology/catalog/speciesbyfamily. Asp. Acesso em fev.

FROESE, R. \& PAULY, D. 2009. FishBase. World Wide Web electronic publication. http://www.fishbase.org/home.htm. Acesso em fev.

FROESE, R. \& PAULY, D. 2010. FishBase. World Wide Web electronic publication. http://www.fishbase.org/home.htm. Acesso em.

GRAÇA, W.J. \& PAVANELLI, C.S. 2007. Peixes da planície de inundação do alto rio Paraná e áreas adjacentes. Maringá. EDUEM. 241 p.

GODINHO, A.L. \& POMPEU, P.S. 2003. A importância dos ribeirões para os peixes de piracema. In: GODINHO, H.P., GODINHO, A.L. (Org.) Águas, peixes e pescadores do São Francisco da Minas Gerais. Belo Horizonte: PUC Minas, p. 361-372.
IUCN Summury statistics 2008 Red list. Disponível em htpp://www. iiucn.org/about/work/programmes/species/red_list/2008_red_list_ summary_statisticsQindex.cfm. Acesso em nov.2008.

KAUFMANN, P.R, LEVINE, P., ROBISON, E.G., SEELIGER, C., PECK, D.V. 1999. Quantifying Physical Habitat in Wadeable Streams. Washington, D. C.: EPA/620/R-99/003, U.S. Biodiversity Environmental Protection Agency, $102 \mathrm{p}$.

KAVALCO, K.F. \& PAZZA R. 2007. Aspectos biogeográficos de componentes da ictiofauna da América Central. ConScientia e Saúde 6(1):147-153.

LANGEANI, F., CASTRO, R.M.C., OYAKAWA, O.T., SHIBATTA, O.A., PAVANELLI, C.S., CASATTI, L. 2007. Diversidade da ictiofauna do alto Rio Paraná: composição atual e perspectivas futuras. Biota Neotropica, Campinas, 5(1):75-78.

LATRUBESSE, E., STEVAUX, J.C., SINHA, R. 2005. Tropical rivers. Geomorphology 70:187-206.

LOWE-McCONNELL, R.H. 1987. Ecological studies in tropical fish communities. Cambridge: Cambridge Univ. Press, $382 \mathrm{p}$.

MACHADO, A.B.M., MARTINS, C.S., DRUMMOND, G.M. 2005. Lista da fauna brasileira ameaçada de extinção: incluindo as espécies quase ameaçadas e deficientes em dados. Belo Horizonte: Fundação Biodiversitas.

PAVANELLI, C.S. \& CARAMASCHI, E.P. 1997. Composition of theichthyofauna of two small tributaries of the Paraná river, Porto Rico, Paraná State, Brazil. Ichthyological Exploration of Freshwaters, München, 8(1):23-31.

REIS, R.E., KULLANDER, S.O., FERRARIS, C.J. 2003. Check List of the Freshwater Fishes of South and Central America. Porto Alegre: EDIPUCRS. 729 p.

SANTOS, G.B. 2010. A ictiofauna da bacia do alto Paraná (rio Grande e rio Paranaíba). MG Biota, 2(6).

SHIBATTA, A.O., GEALH, A.M., BENNEMANN, S.T. 2007. Ictiofauna dos trechos alto e médio da bacia do rio Tibagi, Paraná, Brasil. Biota Neotropica, Campinas, 7(2):125-134. 